\title{
Melan-A/MAGE-3.DP4 Peptide Vaccine
}

National Cancer Institute

\section{Source}

National Cancer Institute. Melan-A/MAGE-3.DP4 Peptide Vaccine. NCI Thesaurus. Code C96041.

A cancer vaccine consisting of a peptide derived from the melanocyte differentiation antigen Melan-A (or MART -1) and the human leukocyte antigen HLA-DP4-restricted human melanoma antigen 3 (MAGE-3.DP4), with potential immunostimulating and antineoplastic activities. Upon administration, Melan-A/MAGE-3.DP4 peptide vaccine may stimulate the immune system to mount a cytotoxic T-cell (CTL) response against tumor cells expressing Melan-A and MAGE-3, resulting in tumor cell lysis. The tumor associated antigens Melan-A and MAGE-3 are overexpressed in a variety of cancer cell types. 